\title{
Doença de Coats : perfil de casos encaminhados para serviço referência em oncologia ocular
}

\author{
Coats Disease: profile of patients referred to \\ a reference ocular oncology service
}

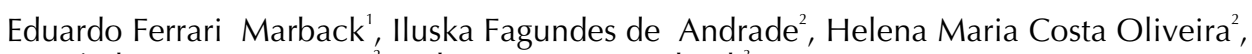
Aristóteles Gazineu Junior ${ }^{2}$, Roberto Lorens Marback ${ }^{3}$

\section{Resumo}

Objetivo: Relatar o perfil de pacientes com doença de Coats encaminhados para afastar o diagnóstico de retinoblastoma. Métodos: Realizado estudo retrospectivo, com base na revisão de prontuários e exames de imagem, de dez pacientes consecutivos. Foram avaliados os dados referentes à idade, sexo, queixa principal, estágio da doença à apresentação, tratamento e evolução. Resultados: A média de idade à apresentação foi de 43,9 meses. Oito pacientes eram do sexo masculino. Leucocoria ou estrabismo foram as principais queixas, presentes em nove casos. Oito pacientes tinham doença em estágio 3B, um estágio 3A e um estágio 2B. Dos oito casos com descolamento total de retina (3B) em sete foi optado apenas pela observação e um submetido a retinopexia sem sucesso. O caso com descolamento parcial (3A) foi tratado por crioterapia e depois feita fotocoagulação por laser diodo, evoluindo com reaplicação da retina e obliteração das teleangiectasias. O caso sem descolamento associado (2B) foi tratado por fotocoagulação por laser de argônio, com boa obliteração dos vasos anormais. A acuidade visual final foi de percepção luminosa em 3 e ausência de percepção luminosa em 5 dos 8 casos com doença $3 \mathrm{~B}$, os casos com doença $3 \mathrm{~A}$ e $2 \mathrm{~B}$ mantiveram visão de movimentos de mão. Nenhum dos casos evoluiu para glaucoma neovascular no segmento médio de 26,7 meses. Conclusão: A maioria dos pacientes com doença de Coats encaminhados para afastar a suspeita de retinoblastoma apresenta doença avançada ao diagnóstico. Nestes casos, devido ao péssimo prognóstico visual e alta complexidade do tratamento cirúrgico, pode-se optar apenas pela observação.

Descritores: Descolamento de retina/etiologia; Vasos retinianos/anormalidades; Glaucoma neovascular

\footnotetext{
'Oftalmologista do Hospital Universitário Prof. Edgard Santos, Faculdade de Medicina da Universidade Federal da Bahia - UFBA e do Hospital São Rafael, Fundação Monte Tabor, Salvador (BA), Brasil;

${ }^{2}$ Alunos do Curso de Especialização em Oftalmologia da Faculdade de Medicina da Universidade Federal da Bahia - UFBA - Salvador (BA), Brasil;

${ }^{3}$ Professor Titular da Faculdade de Medicina, Universidade Federal da Bahia - UFBA e Chefe do Serviço de Oftalmologia do Hospital São Rafael, Fundação Monte Tabor, Salvador (BA), Brasil.
} 


\section{INTRODUÇÃO}

A doença de Coats é congênita, idiopática e não hereditária. Caracteriza-se por telangiectasias retinianas e intensa exsudação. Mais freqüentemente afeta crianças do sexo masculino, na primeira década de vida. Quase exclusivamente a doença é unilateral e raramente acomete adultos ${ }^{(1-6)}$.

Os principais achados clínicos são leucocoria, diminuição da acuidade visual e estrabismo. Por estes motivos, é um dos principais diagnósticos diferenciais com o retinoblastoma. $\mathrm{O}$ diagnóstico se faz pelo aspecto oftalmoscópico e, em casos nos quais existe extenso descolamento de retina associado, pelas características aos exames de imagem, notadamente pela ecografia ocular $^{(1-4,7)}$

O objetivo deste trabalho é relatar o perfil dos casos de doença de Coats, encaminhados ao Hospital Universitário Professor Edgard Santos e ao Hospital São Rafael, para diagnóstico diferencial com o retinoblastoma.

\section{Métodos}

Durante o período de outubro de 2001 a dezembro de 2005, 10 casos novos, que foram encaminhados ao Hospital Universitário Professor Edgard Santos e ao Hospital São Rafael para afastar retinoblastoma, tiveram diagnóstico de doença de Coats.

O diagnóstico foi feito com base no achado oftalmoscópico de telangiectasias retinianas e no aspecto ecográfico para os casos com extenso descolamento

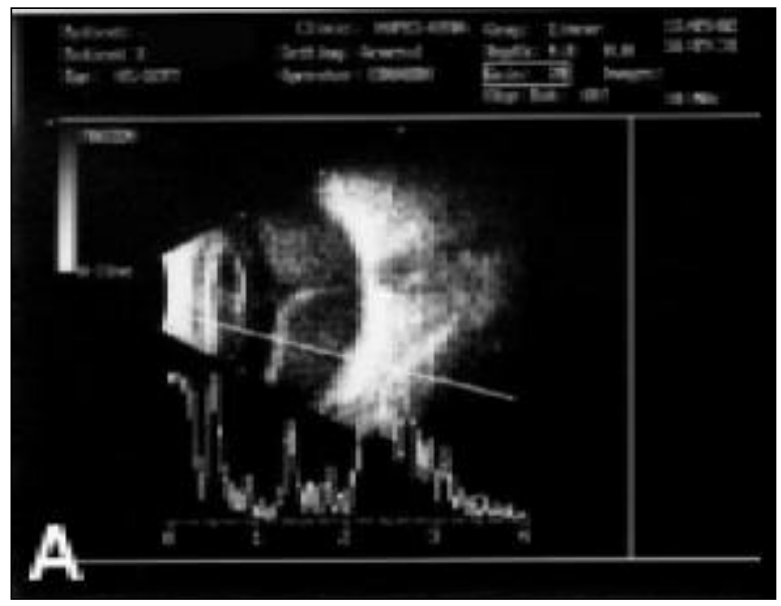

de retina (Figura 1). Os prontuários e exames de imagem disponíveis foram revisados e dados referentes ao sexo, idade, queixa principal, estágio da doença, tratamento utilizado e evolução durante o tempo de acompanhamento foram avaliados.

O estágio da doença, conforme a classificação proposta por Shields e colaboradores, orientou a conduta na maioria dos casos. Esta classificação divide a doença em 5 estágios: 1 - Telangiectasia; 2- Telangiectasia e exsudação (A sem envolvimento macular; B com envolvimento macular);3-Descolamento de retina sem glaucoma (A descolamento subtotal; B descolamento total); 4 - Descolamento total de retina com glaucoma; 5 - Olho cego não doloroso ${ }^{(4)}$.

\section{Resultados}

As características dos 10 pacientes com doença de Coats, e a conduta adotada encontram-se na Tabela 1. No mesmo período, 37 novos casos de retinoblasoma foram diagnosticados. Oito casos eram do sexo masculino e 02 do feminino. A idade média ao diagnóstico foi de 43,9 meses (variando de 11 a 108 meses). Todos os casos tiveram apenas um olho afetado. A queixa principal foi estrabismo em cinco casos, leucocoria em três, leucocoria e estrabismo em um caso e baixa da acuidade visual em outro caso.

Oito pacientes foram classificados como estágio 3B, um estágio 3A e um estágio 2B. Dos casos classificados como 3B, sete foram orientados a manter observação periódica e um foi submetido a retinopexia sem sucesso (Tabela 1). O tempo médio de acompanhamen-

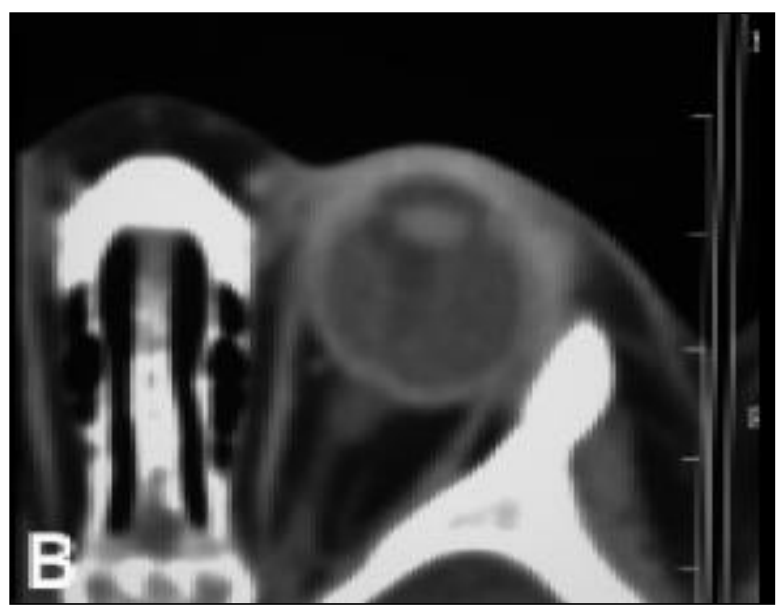

Figura 1: A) Ecografia em corte axial: Caso 2 - Imagem sugestiva de descolamento total de retina, com presença de cristais em líquido subretiniano e ausência de tumor; B) Tomografia computadorizada das órbitas: Caso 10 - Imagem sugestiva de descolamento total de retina, não se vê calcificação ou tumor intra-ocular 
Tabela 1

Características dos pacientes

\begin{tabular}{|c|c|c|c|c|c|c|}
\hline Paciente & $\begin{array}{l}\text { Idade } \\
\text { (meses) }\end{array}$ & Sexo & $\begin{array}{c}\text { Acompanhamento } \\
\text { (meses) }\end{array}$ & Queixa Inicial & Estágio & Conduta \\
\hline 1 & 36 & M & 28 & estrabismo & $3 \mathrm{~B}$ & observação \\
\hline 2 & 72 & $\mathrm{~F}$ & 2 & estrabismo & $3 \mathrm{~B}$ & observação \\
\hline 3 & 20 & $\mathrm{M}$ & 25 & leucocoria & $3 \mathrm{~B}$ & observação \\
\hline 4 & 11 & M & 36 & estrabismo & $3 \mathrm{~A}$ & crioterapia e fotocoagulação \\
\hline 5 & 60 & M & 30 & estrabismo & $3 \mathrm{~B}$ & retinopexia \\
\hline 6 & 108 & $\mathrm{~F}$ & 47 & bav & $2 \mathrm{~B}$ & fotocoagulação \\
\hline 7 & 36 & $\mathrm{M}$ & 1 & leucocoria & $3 \mathrm{~B}$ & observação \\
\hline 8 & 48 & $\mathrm{M}$ & 50 & leucocoria & $3 \mathrm{~B}$ & observação \\
\hline 9 & 12 & M & 26 & leucocoria e estrabismo & $3 B$ & observação \\
\hline 10 & 36 & M & 22 & estrabismo & $3 \mathrm{~B}$ & observação \\
\hline
\end{tabular}

to foi de 26,7 meses (variando de 1 a 50 meses). Em todos os casos foi possível conservar o olho com bom aspecto cosmético. Nenhum paciente evoluiu para glaucoma neovascular durante o período de acompanhamento. Embora não tenha sido possível realizar a medida da acuidade visual inicial em todos os casos, a acuidade visual final, excetuando-se os casos 2 e 7 que tiveram tempo de acompanhamento muito curto, foi de percepção luminosa em 3 casos, ausência de percepção luminosa em 5 casos e visão de movimento de mãos nos casos 4 e 6.

O caso 4 que apresentava doença no estágio $3 \mathrm{~A}$ foi submetido inicialmente a crioterapia e após reaplicação da retina, tratado por fotocoagulação por laser diodo de 810nm (Tabela 1), 36 meses após o tratamento inicial apresentava retina aplicada, sem teleangiectasias aparentes, porém com cicatriz macular exibindo características ecográficas sugestivas de calcificação e visão de vultos. O caso 6 apresentava doença no estágio $2 \mathrm{~B}$, cfoi tratado por fotocoagulação com laser de argônio (Figura 2), evoluiu com resolução da exsudação, manutenção da escara macular após 47 meses de acompanhamento e visão de conta dedos a 1 metro.

\section{Comentários}

A doença de Coats permanece como importante diagnóstico diferencial do retinoblastoma. No mesmo período, tivemos 37 novos casos de retinoblastoma, uma proporção de 1 novo caso de doença de Coats para aproximadamente 4 casos de retinoblastoma. Estes dados diferem da série publicada por Shields et al. avaliando 500 casos de suspeita de retinoblastoma, na qual a proporção entre o diagnóstico de doença de Coats e retinoblastoma foi de 1 para $8,4^{(7)}$. Este maior número

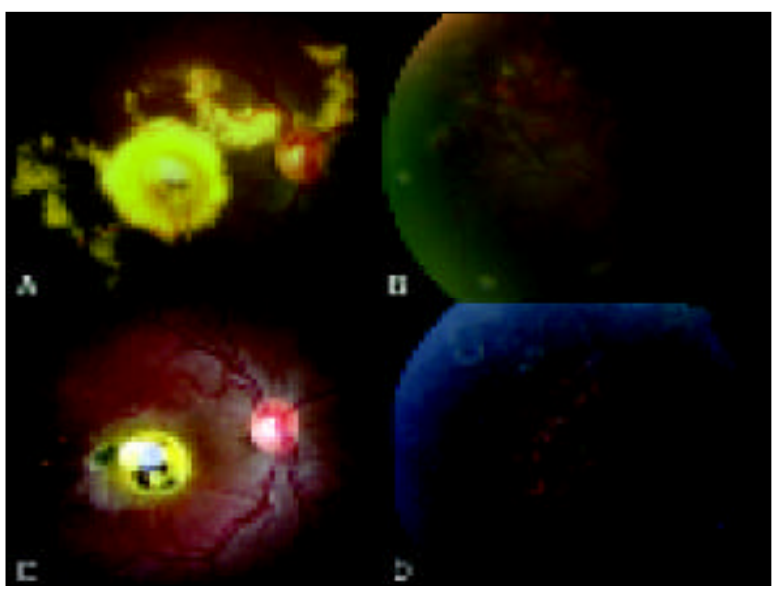

Figura 2: Retinografia, Caso 6. A e B) Aspecto à apresentação com exsudação elevando área macular e evidência teleangiectasias e embainhamento vascular em periferia; C e D) Aspecto 47 meses após a fotocoagulação. Notar lesão macular residual

relativo de casos de doença de Coats encaminhados ao nosso serviço para afastar retinoblastoma, talvez reflita ainda a dificuldade do diagnóstico clínico feito pelo oftalmologista que realizou o atendimento primário, portanto enfatizando a necessidade de se abordar o assunto.

Na nossa série, leucocoria ou estrabismo estavam presentes à apresentação em 9 dos 10 casos, tais queixas também são as mais freqüentemente encontradas no retinoblastoma ${ }^{(1,5,8)}$. Outros achados compartilhados entre as duas doenças incluem o glaucoma neovascular que acontece em $10 \%$ dos casos e, menos freqüentemente, a heterocromia de íris. Mais raramente, podemos encontrar a presença de cristais de colesterol em câmara anterior e hemorragias retinianas ${ }^{(1,5,8)}$. Dentre os dados que ajudam a diferenciar as duas doenças, encontramos a apresentação mais tardia na doença de 
Coats, com idade média que varia de 2,4 a 7 anos e predomínio do sexo masculino sobre o feminino, exibindo uma relação de $3 / 1^{(1,5)}$. Em nossa série, a idade média foi de 43,9 meses, com apenas três casos acometendo crianças menores de dois anos, além disso, houve grande predominância do sexo masculino sobre o feminino com relação de $4 / 1^{(1,5,8)}$. O diagnóstico clínico, pode ser feito com segurança usando estes dados em associação ao achado oftalmoscópico de telangiectasias e exsudação intensa com pontos sub-retinianos brilhantes, que correspondem aos cristais de colesterol identificados à ecografia, além da ausência de tumor sub-retiniano aos exames de imagem ${ }^{(1,5,8)}$.

A etiologia da doença de Coats é desconhecida ${ }^{(1-3)}$. Não existe tendência familiar ou alteração genética comprovada, entretanto a evolução natural da doença segue um curso seqüencial, no qual os vasos retinianos mal formados permitem a passagem e o acúmulo de lipoproteínas na retina, que eventualmente irão romper a membrana limitante externa resultando em descolamento exsudativo. Em sua revisão de 150 casos da doença de Coats, Shields et al. encontraram descolamento exsudativo de retina em $81 \%$ dos pacientes, sendo que descolamento total foi visto em $47 \%$, estes achados foram associados a uma acuidade visual ao diagnóstico menor ou igual a 20/200 em 76\% dos pacientes ${ }^{(5)}$.

O objetivo do tratamento da doença de Coats é destruir as telangiectasias, facilitando a resolução da exsudação, do descolamento de retina e desta forma impedindo a progressão para glaucoma neovascular. Dentre as opções terapêuticas, encontramos a crioterapia, diatermia, fotocoagulação, drenagem de fluido subretiniano, retinopexia e vitrectomia. Usualmente, sendo necessária a associação de mais de uma modalidade terapêutica em múltiplas sessões de tratamento ${ }^{(1,2,4) 5}$. O resultado visual é geralmente pobre; Char estudando 10 casos com acompanhamento médio de 8,8 anos relata acuidade visual final melhor que 20/400 em apenas 2 pacientes ${ }^{(2)}$. Adam et al. tratando 10 pacientes, também só conseguiram manter acuidade visual que permitisse deambulação em 4 pacientes, enfatizando só ser possível este resultado nos casos em que o descolamento de retina não for total à apresentação ${ }^{(9)}$. Já Shienbaum e Tasman conseguiram visão igual ou melhor que 20/30 em 3 de 13 casos e abaixo de 20/200 em oito ${ }^{(3)}$. Aparentemente, estes autores lidaram com casos menos avançados da doença, tal fato pode ser evidenciado pelas modalidades terapêuticas empregadas ${ }^{(3)}$. A maioria dos pacientes daquela série foi tratada por laser ou crioterapia, o que sugere doença no máximo em estágio 3A. Nos três casos em que foi necessária retinopexia, um necessitou ser enucleado, o segundo teve como resultado a ausência de percepção luminosa e o terceiro manteve visão de 20/25, porém este aparentemente não apresentava doença em estágio avançado, uma vez que a acuidade visual inicial era de 20/25 ${ }^{(3)}$. Parece ainda haver uma relação entre o prognóstico e a idade à apresentação, sendo que os pacientes mais jovens geralmente apresentam doença em estágios mais avançados, resultando em acuidade visual final mais baixa ${ }^{(10)}$. Séries provenientes de serviços de oncologia ocular geralmente são formadas por casos encaminhados para se afastar o diagnóstico de retinoblastoma, estes tendem a apresentar baixa média de idade ao diagnóstico, doença em estágios tardios e, portanto, pior prognóstico visual ${ }^{(2,4)}$. Este achado fica explícito na nossa série, onde apenas um entre 10 pacientes não apresentava retina descolada ao diagnóstico (Tabela 1). Outro dado que merece comentário é a nossa opção por não tratar sete dos nossos dez casos. Esta opção, também relatada com certa freqüência por outros autores, foi feita com base nos péssimos resultados visuais, relatados com o tratamento para casos tão avançados, bem como na complexidade e morbidade potencial do tratamento necessário que pode envolver a necessidade de retinopexia e vitrectomia, muitas vezes associadas e repetidas, e sempre sob anestesia geral ${ }^{(1-}$ ${ }^{3,5,10)}$. Já nos dois casos em que não havia descolamento total de retina, conseguimos boa obliteração dos vasos anômalos com tratamento focal por crioterapia e fotocoagulação, embora não tenhamos dados referentes à acuidade visual inicial, pois algumas das crianças não informavam bem, estes dois pacientes foram os únicos que mantiveram visão melhor que percepção luminosa. Até o momento, nenhum dos nossos casos evoluiu para glaucoma neovascular, embora este seja um risco real que temos monitorado.

Após o diagnóstico de doença de Coats, é importante conscientizar os pais da importância do acompanhamento seriado. Embora estas crianças geralmente tenham boa visão no olho contralateral e, portanto, usualmente desempenhem normalmente suas atividades, recidiva tardias podem acontecer. Shienbaum e Tasman relatam em um acompanhamento a longo prazo, recidiva em quatro de doze pacientes tratados, com tempo médio 4,3 anos entre o tratamento e a primeira recidiva. ${ }^{(3)}$ Os mesmos autores enfatizam ainda que dos quatro pacientes com recidiva da doença, três o fizeram em mais de um episódio $^{(3)}$. Tais achados são importantes, pois embora regressão espontânea da doença com reaplicação da retina tenha sido descrita, existe risco de progressão para glaucoma neovascular, especialmente em olhos não acom- 
panhados ${ }^{(1,2,5,11)}$. Nos casos em que o olho torna-se cego e doloroso, a enucleação pode ser necessária ${ }^{(1,2,5)}$.

Em suma, nossos dez casos de doença de Coats, todos encaminhados para afastar o diagnóstico de retinoblastoma, refletem a experiência de outros serviços de referência em oncologia ocular com esta doença $^{(1,2,5)}$. Tratam-se de pacientes com doença em estágios avançados e prognóstico visual reservado. Nos casos em que a retina não apresentava descolamento total, foi possível conseguir bom controle da doença com o uso de tratamento destruidor focal como a crioterapia e a fotocoagulação. Para casos com doença em estágio 3B ou pior, sugerimos individualizar a indicação de tratamento uma vez que este tem alto custo, é complexo, tem possível morbidade associada e resultados pouco encorajadores até o momento.

\section{Abstract}

Purpose: To report the profile of Coats' disease patients, referred to exclude retinoblastoma. Methods: Retrospective study of medical charts from ten consecutive patients. Data evaluated include age at diagnosis, gender, chief complain, disease stage, treatment and evolution. Results: Mean age at diagnosis was 43.9 months. Eight patients had stage $3 B$ of the disease, one stage $3 A$ and one $2 B$. From the eight patients with total retinal detachment (3B disease), seven were managed by observation one was submitted to unsuccessful retinopexy. The patient with partial retinal detachment ( $3 A$ ) was treated with cryotherapy followed by diode laser photocoagulation with resolution of the retinal detachment and obliteration of the abnormal vessels. The patient without retinal detachment (2B), was treated by argon laser photocoagulation with good obliteration of the abnormal vessels. Final visual acuity was light perception in 3 and no light perception in 5 out of the 8 patients with $3 B$ disease, patients with $3 A$ and $2 B$ disease, retained hand motions vision. None of the patients developed neovascular glaucoma in a mean follow-up of 26.7 months. Conclusion: The majority of Coats' disease patient referred to exclude retinoblastoma diagnosis, have advanced disease. In these cases, due to the very poor visual prognosis and high complexity of surgical treatment, simple observation can be an option.

Keywords: Retinal detachment/etiology; Retinal vessels/abnormalities; Glaucoma, neovascular

\section{ReferÊnCIAS:}

1. Shields JA, Shields CL. Review: coats disease: the 2001 LuEsther T. Mertz lecture. Retina. 2002; 22(1):80-91. Review.

2. Char DH. Coats' syndrome: long term follow up. Br J Ophthalmol. 2000; 84(1):37-9.

3. Shienbaum G, Tasman WS. Coats disease: a lifetime disease. Retina. 2006; 26(4):422-4.

4. Shields JA, Shields CL, Honavar SG, Demirci H, Cater J. Classification and management of Coats disease: the 2000 Proctor Lecture. Am J Ophthalmol. 2001; 131(5):572-83.

5. Shields JA, Shields CL, Honavar SG, Demirci H. Coats disease. Clinical variations and complications of Coats disease in 150 cases: the 2000 Sanford Gifford Memorial Lecture. Am J Ophthalmol. 2001; 131(5):561-71.

6. Smithen LM, Brown GC, Brucker AJ, Yannuzzi LA, Klais CM, Spaide RF. Coats' disease diagnosed in adulthood. Ophthalmology. 2005; 112(6):1072-8.

7. Shields JA, Parsons HM, Shields CL, Shah P. Lesions simulating retinoblastoma. J Pediatr Ophthalmol Strabismus. 1991; 28(6):338-40.

8. Marback EF. Tumores do olho e seus anexos. In: Bicas HEA, organizador. Oftalmologia básica. São Paulo: Tecmed e Novo Conceito; 2006. cap. 6. p. 480-97.

9. Adam RS, Kertes PJ, Lam WC. Observations on the management of Coats' disease: less is more. Br J Ophthalmol. 2007; 91(3):303-6.

10. Lai CH, Kuo HK, Wu PC, Kuo ML, Chen YJ. Manifestation of Coats' disease by age in Taiwan. Clin Experiment Ophthalmol. 2007; 35(4):361-5.

11. Wolfe JD, Hubbard GB 3rd. Spontaneous regression of subretinal exudate in coats disease. Arch Ophthalmol. 2006; 124(8):1208-9.

ENDEREÇO PARA CORRESPONDÊNCIA:

Eduardo F. Marback

R. Rodrigo Argolo, 89 - Apt $^{\circ} 201$

Rio Vermelho - Salvador - BA

CEP 41940-220

Email: marback@ufba.br 